\title{
Effective Implementation techniques in Offline Signature Verification
}

\author{
${ }^{1}$ MettaMadhavi, ${ }^{2}$ Manoj Reddy Yaram, ${ }^{3}$ Dr.R.V.Krishnaiah \\ ${ }^{I}$ M.Tech,Department of CSE,D.R.K. college of engineering and technology, Hyderabad, India. \\ ${ }^{2}$ M.Tech,Department of CS,D.R.K. Institute of Science and Technology, Hyderabad, India. \\ ${ }^{3}$ Principal,Department of CSE,D.R.K. Institute of Science and Technology, Hyderabad, India.
}

\begin{abstract}
Handwritten signature verification has long been in use for human identity verification in banks, offices etc. There are both online signature verification techniques as well as offline signature verification techniques. Each technique has its own advantages and disadvantages. This paper compares the results of implementation of three simple offline signature verification techniques. These three filtering techniques are simple mask filtering technique, Local binary pattern and Local Directional pattern. In all these techniques, the original image will be converted to a grayscale image by eliminating the hue and saturation information while retaining the luminance. Next score values will be calculated using each of these techniques. A simple local database of various kinds of signatures is used. A comparison is made based on the scores to evaluate the accuracy of each of these techniques.
\end{abstract}

Keywords:Boundary Extraction;Grayscale;MeanFilter;Offlinesignature;Local Binary Pattern; Local Directional Pattern

\section{INTRODUCTION}

Nowadays, identification and verification is vital in security and resource access control. An interesting method for identification and verification is biometric approach. Biometric is a measure of identification or verification that is unique for a person. Always biometric is carried along with person and cannot be forgotten. Handwritten signature is one of the oldest biometric approaches.

Biometrics can be classified into two categories namely behavioural (signature verification, keystroke dynamics, etc.) and physiological (iris, face, voice characteristics, fingerprint, etc.). Off-line signature verification is considered as a behavioural characteristic based biometric trait in the field of security and prevention of fraud. A significant amount of work on offline signature recognition is available to detect forgeries and to reduce identification errors.

Handwritten signatures occupy a very special place. Verification by signature analysis requires no invasive measurements. Two major methods of signature verification are off-line method and on-line method. On-line method uses an electronic tablet and a stylus connected to a computer to extract information about a signature and takes dynamic information like pressure being applied on pen or paper, the speed at which the signature being put etc. Off-line method uses an optical scanner to obtain hand writing data from a signature written on paper. Off-line systems have an advantage since they do not require access to special processing systems. Offline systems are more applicable and easy to use in comparison with on-line systems. Off-line signature verification compares two signatures after they have been put, using the scanned images of the two signatures as input. Although some information may be lost, the more number of application to which this method can be put more than compensates for its drawback.

There are two main approaches for off-line signature verification namely static approach which involves geometric measures and pseudo dynamic approach which tries to estimate dynamic information from the static image. In off-line systems the objective is to detect three types of forgeries, which is related to intra and inter-personal variability. The first type, called random forgery, is usually represented by a signature sample that belongs to different writer of the signature model. The second one, called simple forgery, is represented by a signature sample with the same shape of the genuine writer's name. The last type is the skilled forgery, represented by a suitable imitation of the genuine signature model.

A signature is a unique identity of a person. No two signatures can be identical, unless one of them is a forgery or a copy of the other. This gives rise to intrapersonal variation, that is, the variation within genuine signature samples of the same individual. At the same time, we also need to consider another type of variability, which is variation between genuine signature samples of two different individuals.

The remaining paper is organized as follows: Section 2 represents the overall architecture of our implementation. Section 3 details on the local signature database. Section 4 describes Gray Scaling. The next three sections discuss on the three types of filtering techniques namely simple mask, LBP and LDP. Section 8 
describes boundary extraction and Section 9 on computation of scores. Section 10 describes our observations. Finally we conclude followed by references.

\section{ARCHITECTURE}

Figure 1 is the Architecture of Effective implementation techniques in offline signature verification

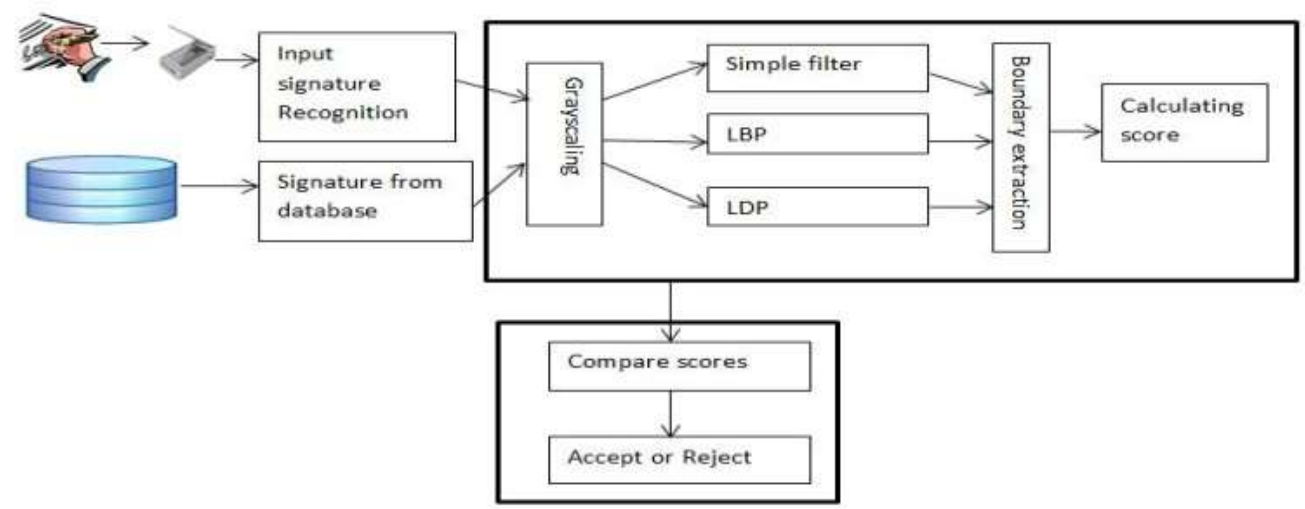

Figure. 1

\section{CREATION OF LOCAL Signature database}

Signature samples are collected from multiplepeople on A4 sheets in predefined space. Each person is requested to sign ten times on the sheet. Forgery signature samples are also collected. These sheets are later scanned and all signatures for each person are cropped, separated and stored in the database along with the signer's name.Instead of searching all the signatures we use signer's name to optimise the search which improves performance.

\section{GRAY SCALING}

Signature image consists of pixels and each pixel has RGB values which mean each pixel is made of red, green and blue color. By combining these we can get a new color. Each color has its value or intensity. These values lie in the range 0 to 255 .

For signature verification, the colour of ink has no significance at all. Instead, the form of two signatures must be compared. Hence all scanned images will be converted to grayscale mages. A grayscale is an image in which the value of each pixel is a sample, that is, it varies only intensity information. Images of this type are known as black and white, are composed exclusively of shades of gray, varying from black at the weakest intensity to white at the strongest. Grayscale images are distinct from one-bit bi-tonal black and white images, which are images with only two colors, black and white (binary images). Grayscale images have many shades of gray in between.

We use the below simple formula to convert RGB images to grayscale by eliminating the hue and saturation information while retaining the luminance.

Grayscale Value $=0.3 *$ red $+0.59 *$ green $+0.11 *$ blue.

\section{FILTERING WITH SIMPLE MASK}

Different types of noises are possible in a signature image (stray dots arising in the image due to improper scanning). To remove noise and improve visual quality of the signature, we apply mean filter on image which is also called nine-point smooth. To apply filter we need a mask. This mask can be a $3 * 3$ matrix, a $5 * 5$ matrix or a $7 * 7$ matrix and so on. In mean filter,asthe mask size increases, the image gets blurred.

To apply mean filter we take a mask of $3 * 3$. In this mask, we initialize all the matrix values with 1.Fig 2 arean example of simple Filter.
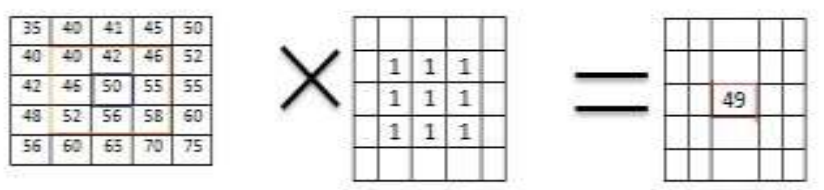

Fig. 2. 
On the left are the image pixel values. Each pixel is marked with its values. The initial pixel value 50 has a dark border and the square area has orange border.All the pixels of the area are multiplies with corresponding mask value. Add them and divide it by 9 (average) and we will get a value 49 .

$((40 * 1)+(42 * 1)+(46 * 1)+(46 * 1)+(50 * 1)+(55 * 1)+(52 * 1)+(56 * 1)+(58 * 1)) / 9=49$

This process will be repeated for all the pixels of the signature image.For pixels that are outside the bounds of the image, we use zero padding. This filter is computationally simple and easy to implement. But if there is more noise, LBP(Local Binary Pattern) is useful.

\section{Filtering With Local Binary PatTern}

The Local Binary Pattern (LBP) operator is defined as gray level texture measure in a local neighbourhood. The most important property of the LBP operator is its invariance against monotonic gray level changes. Equally important is its computational simplicity. LBP operator describes the surroundings of a pixel. Each ILBP(x, y) code is worked out as follows: the eight neighbouring pixels are binarized using as threshold the center gray level value $\mathrm{I}(\mathrm{x}, \mathrm{y})$, generating a binary 1 if the neighbour is greater than or equal to the center; otherwise it generates a binary 0 . The eight binary number are represented by 8 -bit number and saved in $\operatorname{ILBP}(\mathrm{x}, \mathrm{y})$, the range which is $0 \leq \operatorname{ILBP}(\mathrm{x}, \mathrm{y}) \leq 255$

$$
\begin{aligned}
& I_{L B P}(x, y)=\sum_{n=0}^{\prime} s\left(I_{N}(n)-I(x, y)\right) \cdot 2^{n} . \\
& \text { where } s(l)=\left\{\begin{array}{ll}
1 & l \geq 0 \\
0 & l<0
\end{array}\right. \text { the unit step function }
\end{aligned}
$$

Figure 3 shows an example of LBP operator

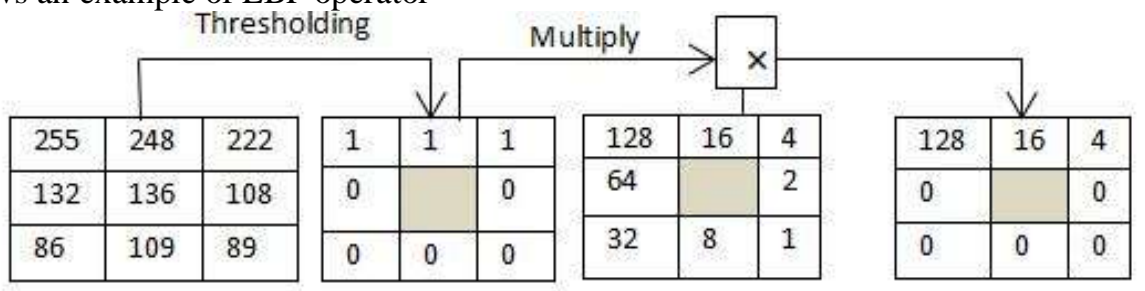

Figure. 3

Figure.3.Calculation of the $\operatorname{LBP}$ code $\operatorname{ILBP}(\mathrm{x}, \mathrm{y})$. In this case $\mathrm{I} \quad(\mathrm{x}, \mathrm{y})=136 . \operatorname{IN}(\mathrm{n})=$ $\{89,108,222,109,248,86,132,255\}, \operatorname{IN}(n)>I(x, y)=\{0,0,1,0,1,0,0,1\}$, so ILBP $(x, y)=4+16+128=148$.

LBP can be extended to a generalized gray level and rotation invariant operator. The major problem of LBP is its sensitivity to the presence of noise and pen dependence. All users have to use same pen because LBP is more able to capture the personal ink distribution when all users use the same pen. But when the personal ink distribution includes pen variability, Local Directional Pattern (LDP) is more convenient.

\section{Filtering With Local Directional Pattern}

The Local Direction Pattern (LDP), transforms the input image I(x, y) to ILDP(x, y), $2 \leq \mathrm{x} \leq \mathrm{N}-1$, $2 \leq y \leq M-1$, Where ILDP $(x, y)$ represents the edge response value of $I(x, y)$ in different directions. Concisely calculates eight directional edge response values for $\mathrm{I}(\mathrm{x}, \mathrm{y})$ using Kirch masks in eight different orientations.

\section{KIRCH COMPASS MASKS}

The masks are defined by taking a single mask and rotating it to eight major compass orientations: North, Northwest, West, Southwest, South, Southeast, East and Northeast

The masks are defined as follows:

The edge magnitude is defined as the maximum value found by convolution of each mask with the image. The direction is defined by mask that produces the maximum magnitude.

$$
\begin{aligned}
& M_{0}=\left[\begin{array}{ccc}
-3 & -3 & -3 \\
-3 & 0 & 5 \\
-3 & 5 & 5
\end{array}\right] \\
& M_{2}=\left[\begin{array}{ccc}
-3 & -3 & -3 \\
5 & 0 & -3 \\
5 & 5 & -3
\end{array}\right] \\
& M_{1}=\left[\begin{array}{ccc}
-3 & -3 & -3 \\
-3 & 0 & -3 \\
5 & 5 & 5
\end{array}\right] \\
& M_{3}=\left[\begin{array}{ccc}
5 & -3 & -3 \\
5 & 0 & -3 \\
5 & -3 & -3
\end{array}\right] \\
& M_{4}=\left[\begin{array}{ccc}
5 & 5 & -3 \\
5 & 0 & -3 \\
-3 & -3 & -3
\end{array}\right] \\
& M_{5}=\left[\begin{array}{ccc}
5 & 5 & 5 \\
-3 & 0 & -3 \\
-3 & -3 & -3
\end{array}\right] \\
& M_{6}=\left[\begin{array}{ccc}
-3 & 5 & 5 \\
-3 & 0 & 5 \\
-3 & -3 & -3
\end{array}\right] \\
& M_{7}=\left[\begin{array}{ccc}
-3 & -3 & 5 \\
-3 & 0 & 5 \\
-3 & -3 & 5
\end{array}\right]
\end{aligned}
$$

Example, mask M0 corresponds to a vertical edge, while mask M5 corresponds to a diagonal edge. The edge responses $\mathrm{ml}, 0 \leq 1 \leq 7$, which represent the edge significance in its respective directions, is worked out as: 


$$
\begin{gathered}
m_{i}=\sum_{i=0}^{2} \sum_{j=0}^{2} l(x-1+i, y-1+j) \cdot M_{i}(i, j) . \\
0 \leq l \leq 7
\end{gathered}
$$

The presence of corners or edges and their orientation will determine the highest response values in each particular direction. $\operatorname{ILDP}(\mathrm{x}, \mathrm{y})$ codes the $\mathrm{k}$ most prominent directions. Hence, the top $\mathrm{k}$ values $|\mathrm{m}|$ are set to 1 and the remainders are set to 0 . The resulting binary number represented by an 8-bit code. In the following example we use $\mathrm{k}=3$, so the LDP code is worked out as:

$$
I_{L D P}(x, y)=\sum_{l=0}^{7} s\left(\left|m_{l}\right|-m_{l, 3}\right) \cdot 2^{n}
$$

E.g. $I(x, y)=136$, $\mathrm{m} 0=\mathrm{I}(\mathrm{x}-1, \mathrm{y}-1) \cdot \mathrm{M} 0(0,0)+\mathrm{I}(\mathrm{x}, \mathrm{y}-1) \cdot \mathrm{M} 0(1,0)+\mathrm{I}(\mathrm{x}+1, \mathrm{y}-1) \cdot \mathrm{M} 0(2,0)+\mathrm{I}(\mathrm{x}-1, \mathrm{y}) \cdot \mathrm{M} 0(0,1)+\mathrm{I}(\mathrm{x}, \mathrm{y}) \cdot \mathrm{M} 0(1,1)+\mathrm{I}(\mathrm{x}+1$, $\mathrm{y}) \cdot \mathrm{M} 0(2,1)+\mathrm{I}(\mathrm{x}-1, \mathrm{y}+1) \cdot \mathrm{M} 0(0,2)+\mathrm{I}(\mathrm{x}, \mathrm{y}+1) \cdot \mathrm{M} 0(1,2)+\mathrm{I}(\mathrm{x}+1, \mathrm{y}+1) \cdot \mathrm{M} 0(2,2)$

Where $\mathrm{m}$ is the third highest value of the sequence $\{|\mathrm{m} 0|,|\mathrm{m} 1|,|\mathrm{m} 2|, \ldots,|\mathrm{m} 7|\}$. In case of a tie, which means there are several values equal to $\mathrm{ml}, 3$, the only bit set to 1 is the most significant.

\begin{tabular}{|l|l|l|l|l|l|l|l|l|}
\hline Kirch Mask & $M_{7}$ & $M_{6}$ & $M_{5}$ & $M_{4}$ & $M_{3}$ & $M_{2}$ & $M_{1}$ & $M_{0}$ \\
\hline$m_{f}$ value & -385 & 877 & 2053 & 1333 & 37 & -1131 & -1465 & -1283 \\
\hline Rank & 7 & 6 & 1 & 3 & 8 & 5 & 2 & 4 \\
\hline Code bit & 0 & 0 & 1 & 1 & 0 & 0 & 1 & 0 \\
\hline LDP code & \multicolumn{7}{|c|}{50} \\
\hline
\end{tabular}

Fig.4 . calculation of the LDP code $I_{\text {LDP }}(x, y): I(x, y)=136$

$$
\begin{aligned}
& \left\{\left|m_{i}\right|, O<=\mid<=7=\{1283,1465,1131,37,1333,2053,877,385\}\right. \\
& m_{i, 3}=1333,\left|m_{i}\right|>m_{i, 3}=\{0,0,1,1,0,0,1,0\} \\
& \text { So } I_{L D P}(x, y)=2+16+32=50
\end{aligned}
$$

By using kirch mask formula the values will be calculated for each pixel and these values will be replaced with corresponding pixels values.

\section{BOUNDARY EXTRACTION}

The boundary of each image will beprocessed and identifiedto eliminate blank areas from the sides of the image. This ensures each image consists only of the signature part. We avoid resizing the images to the same width or height as it distorts the image to very large extent, whose effect is even more profound in the transform domain.

\section{COMPUTATION OF SCORES}

After boundary extraction, the input signature will be compared pixel by pixel with each of the signers signatures stored in the database. A percentage score based on the matching red, blue and green values is calculated. If the score is greater than $88 \%$ then the signature will be accepted as genuine. This is repeated for each of the filtering techniques and the scores are observed for accuracy.

\section{OBSERVATION}

The LDPs produces more stable pattern than LBP and Simple mask when there is variability of pen used in the signature. A sample comparison of the three filtering techniques is shown in the below diagrams.

Fig $5 \mathrm{a}$ is the original input signature. Fig $5 \mathrm{~b}$ is the grayscale signature image. Fig $6 \mathrm{a}$ is the filtered signature image using simple filtering (mean filter) and Fig $6 \mathrm{~b}$ is the boundary extracted image of the filtered signature. Fig $6 \mathrm{c}$ shows the final relevant signature retrieved from the database after applying grayscale, simple filter and boundary extraction. Fig $6 \mathrm{~d}$ shows the computed accuracy value.

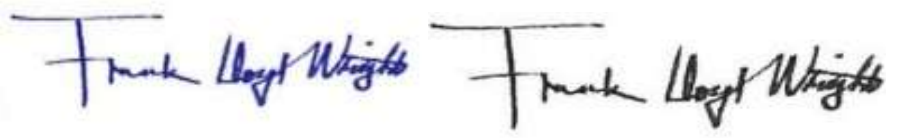

5a.Input signature image 5b.Grayscale signature image

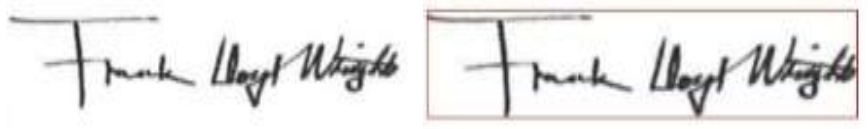

6a.Simple Filtered signature image6b.Boundary extraction of input image 


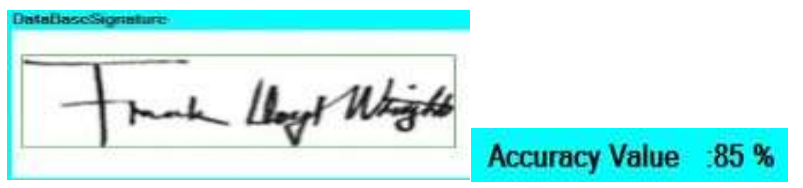

6c.Signature from the database6d. Finding accuracy value

Fig7a is the filtered signature image using LBP and Fig $7 \mathrm{~b}$ is the boundary extracted image of the filtered signature. Fig 7c shows the final relevant signature retrieved from the database after applying grayscale, LBP and boundary extraction. Fig $7 \mathrm{~d}$ shows the computed accuracy value.

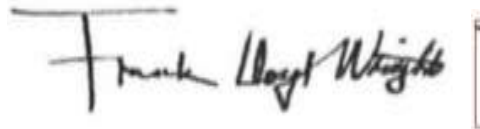

7a.Filtered image using LBP

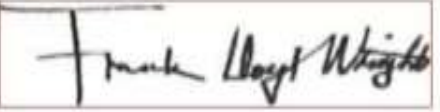

7b.Boundary Extraction

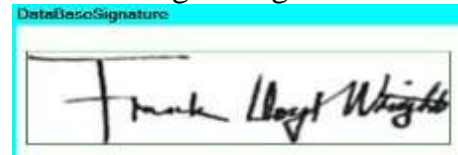

7c.Signature from the database7d.Finding accuracy value

Fig 8a show the filtered image using LDPand Fig $8 b$ is the boundary extracted image of the filtered signature.

Fig $8 \mathrm{c}$ shows the final relevant signature retrieved from the database after applying grayscale, LDP and boundary extraction. Fig 8d shows the computed accuracy value.

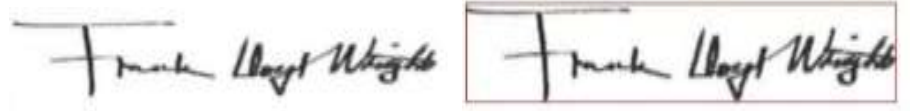

8a.Filtered signature image using LDP8b.Boundary Extraction

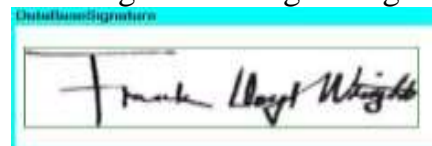

8c.Signature from the database $8 \mathrm{~d}$.Accuracy value of LDP

This is an example of a signature image which is not pen dependent. Hence we can see the accuracy values of simple filtering, LBP and LDP. The score is best in LDP. So LBP is more useful when all the signatures are signed with the same pen and LDPs are more useful when Signatures are signed with different pens.

\section{CONCLUSION}

This paper proposes effective implementation techniques for offline signature verification system. Simple filter which is also known as mean filter is computationally very simple and is useful when the scanned signature is very clear (less noisy). This is more useful in salt pepper noise cases. LBP (Local Binary Pattern) is efficient when there are a group of signatures to be tested which are signed using the same pen and has less presence of noise. If the signature to be tested is signed with different pens then LDP (Local Directional Pattern) is useful. So LDPs give more accurate result than LBPs when there is no pen dependence. LDPs and LBPS are more efficient in detecting skilled forgeries as well as simple and random forgeries.

\section{REFERENCES}

[1] W. Ho, X. Ye and k. Wang. "A survey of off-line signature verification" in International conference on Intelligent Mechatronics and automation.IEEE. August 2004

[2] Meenakshi k. Kalera, "Offline signature verification and identification using distance statistics", International Journal of Pattern Recognition

[3] Prof. PhD. VasileGui, "Smooting Techniques in image Processing",

[4] Meenakshi S Arya, "A Preliminary Study on Various Off-line Hand Written Signature Verification Approaches", International Journal of computer applications 2010.

[5] Miguel A.Ferrer," Off-line Signature Verification using Local Patterns", Telecommunications (CONATEL), 2011 2nd National Conference on Date of Conference: 17-20 May 2011

[6] Dr. Longin Jan Latecki , "Edge detetion", HaoHuy Tran computer graphics and image processing CIS 581-Fall 2002.

[7] Baochang Zhang, YongshengGao, "Local Derivative Pattern versus Local Binary Pattern: Face Recognition with high order local Pattern Descriptor”, IEEE Transactions in image processing, Vol. 19.no.2,February 2010 
[8] DakshinaRanjanKisku,Phalguni Gupta, "Offline signature identification by Fusion of multiple classifiers using statistical learning theory”, International Journal of Security and its applications Vol, 4,No. 3,July,2010

[9] M. Ammar, Y. Yoshida, T. Fukumura, "A new effective approach for automatic off-line verification of signatures by using pressure features", in Proceedings of 8th international Conference on Pattern Recognition, pp. 566-569, 1986

[10] T.Jabid, M.H.Kabir and O. S. Chae, "Local Directional pattern (LDP) for face recognition",in International Conference on CONSUMER Electronics, January 2010.

[11] T. Ojala, M.Pietikainen, T. Maenpaa, "Multiresolution Gray-Scale and rotation invariant texture classification with local binary patterns", IEEE Transactions on Pattern Analysis and Machine Intelligence,Vol.24,no 7,971-987,2002

[12] D. Bertolini,L.S.Oliveira,"Reducing Forgeries in writer independent off-line signature verification through ensemble of classifiers", in Pattern Reognition, vol 43,no. 1,pp.387-396,January 2010.

[13] Tan Yng-Zing 40218409, Prof. Kurt Kubik ,"Offline Signature Verification based on Shape and Density Feature",School of information Technology \& Electrical Engineering.

\begin{tabular}{|l|l|} 
& $\begin{array}{l}\text { Author1: MettaMadhavi } \\
\text { B.Tech CSE from VRSEC Vijayawada, Currently pursuing M.Techin Computer Science and Engineering fromDRK } \\
\text { College of Engineeringand Technology,JNTUHyderabad } \\
\text { Her Main Interest includes Image Processing and datamining, DotNet Programming, Networking, Data Structures, } \\
\text { Operating Systems, Compiler Design. }\end{array}$ \\
$\begin{array}{l}\text { Author 2: Manoj Reddy Yaramreceived his B.Tech degree in Computer Science and Engineering from TRR } \\
\text { College Of Engineering, Studies in 2009. He is Certified as IBM "Data Base Associate-DB2 9 Fundamentals". } \\
\text { From 2009 to 2010 he was with the VyshnaviBEd college, Gurazala, Guntur (dist.), AP. Since 2010, he has been an } \\
\text { M.Tech student in Computer Science department in the DRK Institute of Science and Technology. In the mean while } \\
\text { from January 2012, he is working as an Asst. Professor in A.M.REDDY Memorial College Of Engineering and } \\
\text { Technology, Narasaraopet, Guntur (dist.), Andhra Pradesh, India. } \\
\text { His current research interests include Image Processing, Data mining, Networkingand Compiler Design. }\end{array}$ \\
$\begin{array}{l}\text { GuideDr.R.V.KrishnaiahPrincipal } \\
\text { DRK Institute of Science\& Technology,B.Tech in ECE from Bapatla Engineering College, Nagarjuna } \\
\text { UniversityM.Tech in EIE from NIT Warangal former RECM.Tech in CSE from JNTU HyderabadPh.D in ECE from } \\
\text { JNTU AnantapurPresently working as Principal at DRKISTearlier worked in ECIL, Vasavi College of Engg, } \\
\text { VNRVJIET, ChiralaEngg.College, as ProfessorMITS, PIRMEC, Viswabharathi College of Engg. as Principal } \\
\text { Hyderabad. His main research interest includes Data Mining, Software Engineering. }\end{array}$ \\
\hline
\end{tabular}

